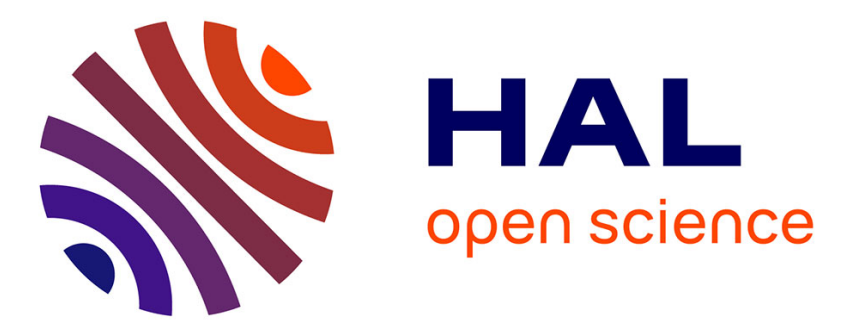

\title{
Creating Smarter Spaces to Unleash the Potential of Health Apps
}

\author{
Jean-Marie Bonnin, Valerie Gay, Frédéric Weis
}

\section{To cite this version:}

Jean-Marie Bonnin, Valerie Gay, Frédéric Weis. Creating Smarter Spaces to Unleash the Potential of Health Apps. ICOST 2018 - 16th International Conference on Smart Homes and Health Telematics, Jul 2018, Singapore, Singapore. pp.134-145, 10.1007/978-3-319-94523-1_12 . hal-01954045

\section{HAL Id: hal-01954045 \\ https://hal.inria.fr/hal-01954045}

Submitted on 13 Dec 2018

HAL is a multi-disciplinary open access archive for the deposit and dissemination of scientific research documents, whether they are published or not. The documents may come from teaching and research institutions in France or abroad, or from public or private research centers.
L'archive ouverte pluridisciplinaire HAL, est destinée au dépôt et à la diffusion de documents scientifiques de niveau recherche, publiés ou non, émanant des établissements d'enseignement et de recherche français ou étrangers, des laboratoires publics ou privés. 


\title{
Creating smarter spaces to unleash the potential of health Apps
}

\author{
Jean-Marie-Bonnin ${ }^{1}$, Valérie Gay $^{2}$, and Frédéric Weis ${ }^{3}$ \\ 1 IMT Atlantique - IRISA, Rennes, France \\ jean-marie.bonnin@irisa.fr \\ 2 University of Technology Sydney, Broadway NSW 2007, Australia \\ valerie.gay@uts.edu.au \\ ${ }^{3}$ University Rennes 1 - IRISA, Rennes, France \\ frederic.weis@irisa.fr
}

\begin{abstract}
Technologies necessary for the development of pervasive health apps with intensive and seamless interactions with their environments are now widely available. Research studies and experimentations have demonstrated the real ability for health apps to interact with their environment. However, designing, testing and ensuring the maintenance and evolution of pervasive health apps remains very complex. In particular, there is a lack of tools to enable developers to design apps that can adapt to increasingly complex and changing environments (sensors added or removed, failures, mobility etc.). This paper reflects our vision to reduce this complexity and is based on our current research work on smart environment and personalized health monitoring apps. It uses SAM, a smart asthma monitoring app as an illustration to highlight the need for a comprehensive set of new interactions to help health app developers interact with the users' environment, and more specifically get a smarter access to the data. Some requirements can be on the minimum quality level of the data and the way to adapt to the diversity of the sources (data fusion / aggregation), on the network mechanisms used to collect the data (network/link level) and on the collection of the raw data (sensors). It discusses possible solutions to address these needs.
\end{abstract}

Keywords: Smart Space, Ubiquitous Applications, Edge/Fog computing, e-Health, Chronic disease management, Asthma Management, IoT, Context awareness

\section{Introduction}

Smart space eco-systems are becoming a mass market reality. Large areas of our living spaces are now instrumented: short/long-range and low energy communications, a broad variety of visible (smart objects) or invisible (sensors and actuators) objects. The applications of the smart spaces are boundless. There is a real ability to adapt the smart space to the behaviors and needs of users, however mainstream applications are barely existent. 
In this paper we use the definition of [8] for smart space: $A$ smart space is a smart environment that has commuting embedded into it and can provide information that can be used to model the real world into the virtual computing world.

This paper envisions smarter spaces with massive but scalable interactions between individuals and their everyday working and living environments such as residential housing, public buildings and vehicles. It focuses on the needs of app developers for better ways to interact with their environment and in particular use the opportunity of tapping into the vast amount of data collected from the physical environments for health apps. Today, this can only be done if the environment has especially been developed for it (for example "smart" hospital room). They are difficult to adapt to increasingly complex situations, even though environments in which they evolve are more open, or change over time (new sensors added, failures, mobility etc.)

Being able to develop health apps that are ready to deploy and evolve in everyday environments should allow significant cost reduction. Unfortunately, designing, testing and ensuring the maintenance as well as the evolution of such a pervasive application remains very complex. In our view, the lack of resources by which data issued from real environments are made available to health app developers is a major concern.

This paper is looking into this lack of data exposure and its objective is to explore how application development, setup and maintenance could be simplified in an open smarter space eco-system. Using an existing health app we developped as a basis, section 2 presents an example on asthma management and shows how this chronic condition would benefit from access to the data issued by the smart space eco- systems. Section 3 gives our motivation for a new approach and focuses on one main challenge: providing health app developers with a better and smarter access to the environment data. The paper concludes with perspectives and future work.

\section{Illustration: SAM - Smart Asthma monitoring}

One chronic condition that would benefit from getting better data from smart space eco-systems is asthma. According to GAN ${ }^{4}$ in 2014, asthma may affect more than 334 million people worldwide. A few projects focusing on Asthma management exist [16] but they either use a tightly controlled environment or they do not adapt to their environment and rely mainly on personal area networks and specialized individual sensors (e.g. peakflows). Some cloud infrastructure solutions exist [7]. However they do not have the level of pervasiveness this paper envisions.

Figure 1 illustrates our vision. It features one citizen, Alex, an active middle age man that is prone to asthma attacks mostly triggered by pollen and pollution and a team of clinicians that help him managing his condition. Alex is using

\footnotetext{
${ }^{4}$ Global Asthma Network
} 
the app we developed. The app collects health and fitness data from different sources and gives Alex the possibility to aggregate data from different sources. The app interacts with a wide range of wireless devices and wearable health trackers and also aggregates data from third party apps. It offers Alex personalized exercise tracking and monitoring of biometric data such as heart rate, respiration, body temperature, weight, food intake, blood pressure, cholesterol, asthma, blood glucose and many more.

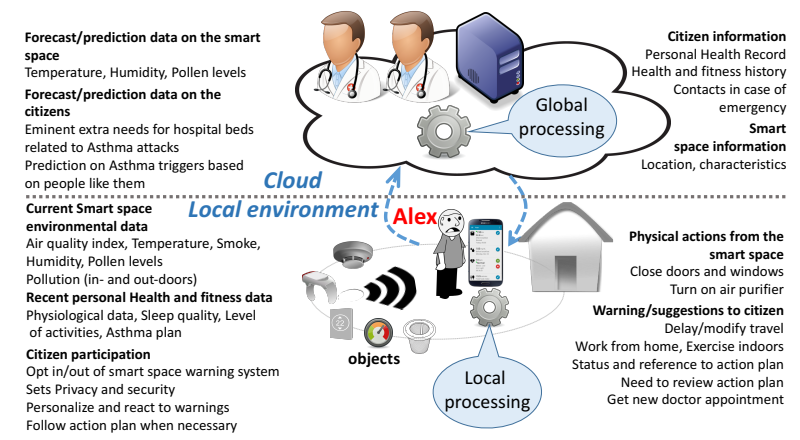

Fig. 1. Illustration of an asthma management scenario

Three important steps are involved in his asthma management in the smart space: Data collection, Data analysis and Nudge, feedback and service presentation.

\subsection{Data collection for asthma}

On a typical day, while Alex is living an active life, the smart space collects as much relevant data as possible in order to offer Alex the best overview on his asthmatic condition. His app collects data from wireless devices supporting open standard protocols such as the Google Android Wear smart watches and fitness trackers that allow third party developers to retrieve the data directly from the device (Figure 2, box 1). It also collects data from devices (peakflow meter, pollution meter) paired with Alex's mobile device (Figure 2, box 2) via Bluetooth or ANT+. Alex is in control of the data he wants to collect and how it is collected. He can enter data manually (Figure 2, box 3) and annotate a reading by adding comments and contextual information, such as extra performed activities. For closed and proprietary wireless devices (Figure 2, box 4 and 5), data is obtained via their server through an open API (e.g Fitbit, Jawbone, Withings, myFitnessPal and Fatsecret).

Data is also collected from the smart space in order to offer more fine-grained analysis (Figure 2, box 6). The context of Alex can be important and one of the challenges is to identify and collect the data that is important for a particular 
user in order to provide timely feedback. For Alex, our app captures locationbased information about the Air Quality Index (AQI) and pollen levels. In the global scenario summarized in Figure 1, our mobile application collects the data within Alex's smart space and in the cloud, for example, to get real-time data on the pollen and pollution levels in the different locations Alex intends to travel to today. Another example is to get forecast on air quality in Alex's smart home environment.

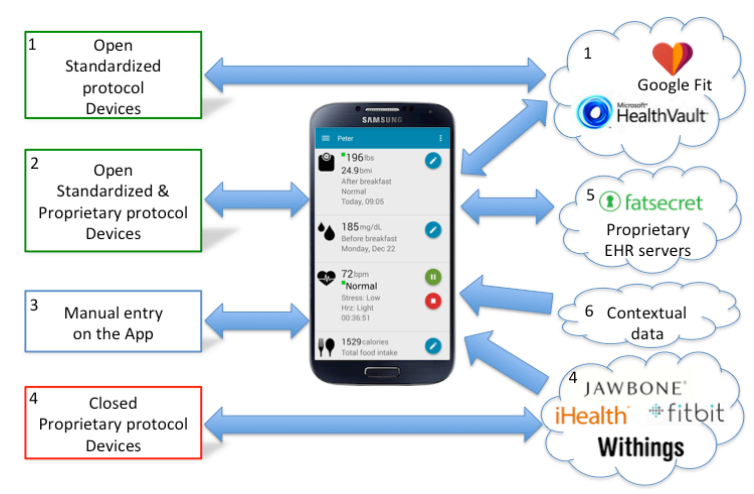

Fig. 2. Our app's eco system

\subsection{Data analysis and data storage for asthma}

Currently, the app stores all data locally on the mobile device, or on the cloud on a server chosen by the user. Alex opts for that in order to get more intelligent feedback based on his long-term history and possibly by comparing his data with the data of other citizens with similar conditions. Some of his sensors can also contribute to crowd sensing and help other asthma sufferers. Alex also opts for his clinicians to get access to his data and decide, for example, that there is a need to make adjustments to his asthma plan. This option gives Alex's clinicians an overall view on his health and fitness data and help them making a more accurate and personalized assessment. This could also allow hospital to estimate how many hospital beds they need to reserve for victims of asthma attack.

\subsection{Nudge, feedback and service presentation}

For the feedback, we opted for a user-centred approach where Alex is in control of the way the data is presented to him. Triggers are personalised to Alex and the app provides threshold to trigger an alert. For example, the app would alert Alex when the air quality deteriorates and gets under his thresholds and raises an alarm if it gets hazardous for his condition. A similar approach will be taken 
for the pollen level. Alex can either see the data in real-time, or just get a notification or nudge when necessary or even ignore the data and get a phone call from his doctor if he needs to do something. Alex opted for a nudge when needed and an automatic reaction from his smart space when possible (automatic closing/opening of doors and windows at home or in the car, adjustment of the air conditioning). He selected to have real-time analysis and get warnings and references to his asthma plan based on his personal threshold levels on several parameters (including pollen and pollution level, peak flow readings). He may also be contacted by his clinicians to make an appointment or to adjust his asthma plan. Based on his calendar that contains the place and activities, he is planning to go to, he may also receive some advice to change plans (e.g.: work from home, exercise indoor) based on the location's current and forecasted data. Alex lives in Australia and he has an asthma action plan: a written plan, prepared for him by a healthcare professional. It allows him to reduce the severity of acute asthma flare-ups. Asthma action plans help recognize worsening asthma and advise what to do in response. They typically include: a list of prescribed medication, including dosage; what to do when asthma gets worse; what to do in an emergency; the name of the health professional predominantly responsible for treating the person and the date. In the future we would like Alex's asthma plan to be integrated in a health applications that would monitor the different triggers in the smart spaces and react according to the patient's asthma plan.

\subsection{Challenges to develop SAM to support this vision}

Most of the vision presented in this example is feasible today but parts of it still bring interesting challenges. This section highlights some of the challenges developers have to be able make this vision possible.

Silos There is an increasing amount of health- and fitness-related information that has been collected and stored in the cloud. However, the data usually resides in silos and in addition, in most cases health and fitness data is separated. According to Mandi et al [11], these data streams will initially remain confined to their respective platforms and will have very limited ways to integrate with electronic health records (EHRs). To provide better health outcomes and better patient engagement, a complete picture is needed which combines informal health and fitness data collected by the user, together with official health records collected by health professionals. Combining these two streams, the data can be analyzed using data analytics and health professional expertise to offer better personalized advice and care. There is good evidence that the integration can improve therapeutic management [3].

For chronic disease patients, there is often a need to monitor several physiological parameters and not just their activity level or environmental data. The presence of data silos prevents these users from obtaining an overall view of their condition. To engage these users in active monitoring of their condition, it is important to have all health and fitness data in one place and give them a personalized overview and feedback on trends and progress. Moreover, device man- 
ufacturers develop their own protocol and data formats to retrieve data from the device or to send commands to the device. Some protocols are straightforward, using plain text to send or receive data. Many however, implemented complex protocols with numerous commands to control and exchange data. Without a detailed specification it is impossible to communicate with these devices.

Quality and privacy of the data With the increase of data sources comes the need to be able to differentiate the sources based on their quality, security and trust levels. There is a need to express requirement on the network mechanisms used to collect the data (network/link level) security aspects in particular - and on the collection of the raw data (sensors). This would lead to a better service for asthma sufferer as their asthma management would be able to dynamically adjust itself to the status of the smart-space (e.g. change the air conditioning at home or in a personal car).

Abstraction level Health app developers also need to get the right level of abstraction to trigger an action. To get to that level of abstraction (e.g. is the pollution level too high wrt the users thresholds) there is a need to combine data at different levels (e.g. raw or elaborated data).

Detection of unreliable sensors The reliability of the sensors varies widely and reliability is very important for health apps like SAM and health professionals that need to make a diagnostic. In Alex's case, air pollution data comes from accurate and expensive sensors like the ones of his high tech building at work, but it also comes from the low cost gadget Alex is carrying. The gadget might be untested and therefore not be fit for purpose to justify, on its own, a change to his medication or his asthma plan. For example, devices made for the fitness market are not necessarily approved by the Food and Drug Administration (FDA) and as such even less reliable. If we are able to tags the source of the reading, it would be beneficial for a health professional in his/her assessment of the data quality. Many health professionals discard self-collected health. Fitness and environmental data due to the unreliability of the data and prefer to use their own data for diagnosis. We believe that over time more health professionals will accept the data if the source is properly tagged so that they know which device, or which application, generated the data.

\section{Smarter access to the local data}

The technologies supporting the pervasive services presented in the asthma management example, with intensive and seamless interactions between individuals and their environments, are now widely available. Spaces in which Alex lives are increasingly enriched with these technologies. Alex's asthma can be monitored in his working and living environment: residential housing, public buildings, vehicles, etc. as illustrated on Figure 3a. These environments are themselves organized in silos that do not interact with each other and do not coordinate 
well. In each environment, designing an application involves implementing one or more logical control loops which include four stages: (1) data collection in the real environment, (2) the (re)construction of information that is meaningful for the application and (3) for decision making, and finally, (4) action within the environment. While many decision-algorithms have been proposed, the collection and construction of a reliable and relevant perception of the environment still poses major challenges.

Developing Health apps that are ready to evolve in different environments and that overcome the existing silos would involve significant cost reduction. Unfortunately, as illustrated in section 2, designing, testing and ensuring the maintenance as well as the evolution of a pervasive health application remains very complex. In our view, the lack of resources by which properties of the real environment are made to application developers is a major issue. Most current solutions are based on a massive collection of raw data from the environment, stored and processed on remote servers [12] and use specialized cloud services for delivering pervasive services. These solutions isolate the developer from the real environment that often needs to be depicted according to complex, heavy and expensive processes.
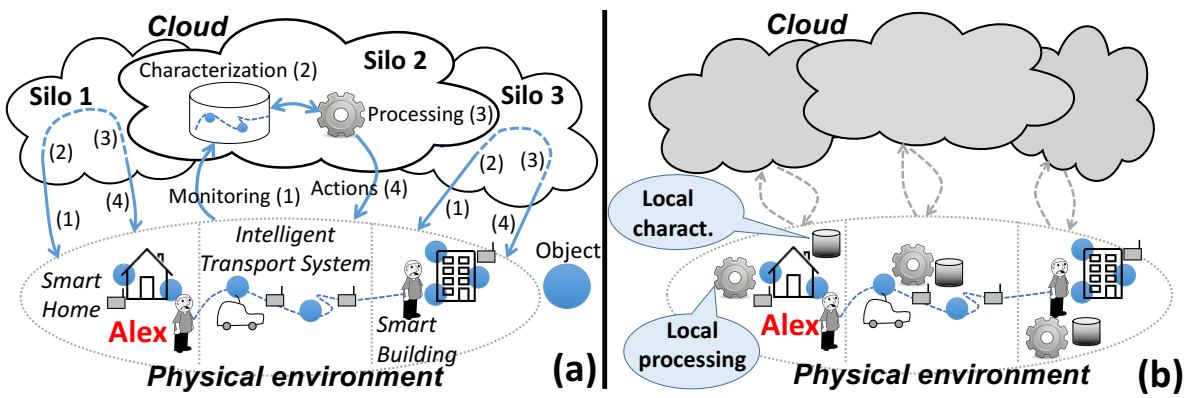

Fig. 3. Design of a pervasive app using cloud services/local data and local processing

However, new paradigms like edge or fog computing [14] offer alternative ways of bringing computing capabilities to the edge of the network. Objects and infrastructure integrated into user smart space often provide a more suitable support to pervasive applications. The objects can carry different kinds of data, like current smart space air quality. The description of the actual state of the environment can be richer, more accurate, and, meanwhile, easier to handle. The processing can be distributed by being built directly into the environment, facilitating scalability and resilience by the processing autonomy; and finally, moving the processing closer to the edge of the network avoids major concerns about data sovereignty and privacy encountered in cloud-based infrastructures. Figures $3 \mathrm{~b}$ and 1 illustrated a plausible "distribution" of an asthma management scenario between Alex's local environment and cloud-based services. The 
best place to use the data depends partly on what kind of data is needed for making decision and trigger processing. Fine decisions can be made closer to the objects producing the data. Local data characterization and local processing reduce the computing and storage resources in the cloud. The latter can be always used to store selected or transformed data for global historical analysis or optimization, for example for health and fitness history.

There is a need for a comprehensive set of new models and architectures to help smart space designers making those distribution choices. We propose a design approaches that exposes local properties of the environment to pervasive applications. The developer must be able to manage and to enrich locally data produced in the environment. In return the application would then be able to build their knowledge about their environment (perception) in order to adjust their behavior (eg. level of automation) to the actual situation. In the following, we present three research directions to develop models of data-centric service architecture.

\subsection{Characterizing data quality}

Several studies have proposed data quality analysis [9] and methodologies for identifying data quality issues [5]. The quality of the raw data collected in the smart space (temperature, sound level, pollen level etc.) are of crucial importance to assess the quality of the perception of the environment and therefore to ensure correct behavior of a health pervasive app. Poor quality data is often a result of poor representation of the physical environment. Data quality can be characterized through several dimensions. Here the term dimension is defined as a "measurable extent of a particular kind": timeliness, accuracy, confidence, reliability, confidentiality, etc. For example, timeliness refers to time data should be available for use by an app within an acceptable time relative to its time of creation.

In an environment instrumented with smart objects and sensors interconnected with low energy communications, the way data is produced, consolidated, and aggregated has an impact on its quality dimensions. Moreover part of these quality dimensions requires gathering information from several communication layers (link and network layers) at several entities (the object itself, access point, router etc.) to be computed on the fly. Lightweight cross-layer interactions to collect relevant data have to be designed for this purpose, the goal is to expose "augmented" data (initial raw data + some pertinent dimensions selected by the app developer) to the pervasive app. Figure 4 illustrates these principles.

A frugality principle should guide the design, therefore it is not appropriate to build all imaginable attributes. It is necessary to identify attributes relevant to the application and to have mechanisms to activate or deactivate at run-time the process to collect them. A "frugal" service uses as few resources as possible, while having the possibility to use much more when it is required. This is quite a different approach than best-effort services where a networked application uses all the resources it can use and if there are not enough resources, it tries to mitigates the effects. 


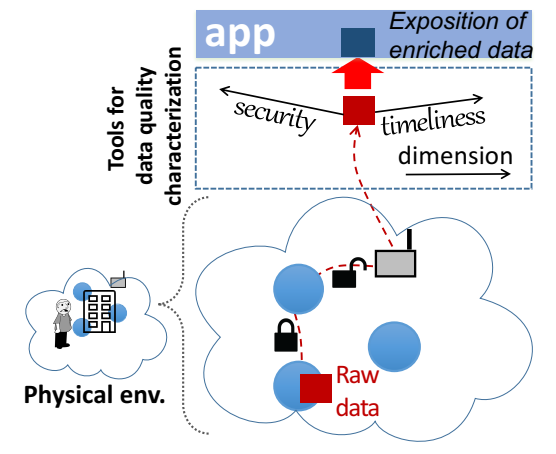

Fig. 4. Enriching data collected in smart environment using cross-layer interactions

\subsection{Providing pertinent abstractions}

Raw data should only be used directly to determine low-level abstractions. Further help in abstracting from low-level details can be provided by data fusion. The pervasive community increasingly understands that developing pervasive apps should be supported by such data fusion tools [15]. While many definitions exist for data fusion, we use the one in [10]: Information fusion is the study of efficient methods for automatically or semi-automatically transforming information from different sources and different points in time into a representation that provides effective support for human or automated decision making.

A good construction of a meaningful information (for example "air quality in the home", "level of activity in a room", "posture of a person" etc.) reduces the complexity and helps developers to concentrate on the application logic.

Moreover, the reactivity required in pervasive systems and the aggregation of large amounts of data (and its processing) in the cloud are antagonists. Fusion tools that can be deployed closer to the edge of the network are needed.

Traditional data fusion systems are often designed to use a set of dedicated sensors. Moreover, most approaches rely directly on sensor measures making them hard to deploy in many different environments and for different pervasive apps. They require an ad hoc tuning phase as the behavior of a sensor can change from one environment to another. Finally, the techniques to be used in our approach are guided by different criteria [13]: (1) the relevance of abstractions produced for pervasive applications, (2) the anonymization of exploited raw data, (3) the processing time and computing power required for processing, (4) the ignorance of the system (the system should be able to be indecisive when it does not have enough clue of what is going on) and (5) the no predetermined sensor configuration (the data fusion process should be if possible independent from the configuration of sensors).

We proved [13], through the use of dedicated algorithms and a layered architecture that data fusion tools can be deployed using the Belief Function Theory (BFT) [6]. Raw data collected in the environment can been seen as an "accumulation of evidence". BFT enables the use of multiple sources to infer a meaningful 
abstraction defined by app developers. In our approach, an abstraction used by pervasive app is computed by a chain of three tasks as illustrated in Figure 5:

- Model. The transition between a raw sensor value and a model of the sensor is made through the use of a "belief function" which maps each sensor value to a possible state (state 1 , state 2 etc.) of the abstraction. A belief function represents the "degree of belief" associated to each possible value of the abstraction.

- Fusion. Then a set of belief functions (corresponding to a set of sensors) can be combined (fused).

- Decision. Finally the system can decide what is the "best" value for the abstraction.

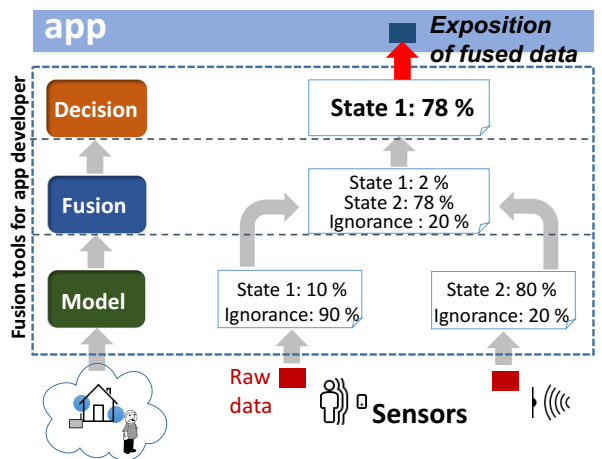

Fig. 5. Building high level abstraction with raw data collected in smart environment

\subsection{Detecting new sensors / detecting unreliable sensors}

One of the reasons why pervasive apps are not affordable for mainstream adoption comes from the necessity to control all the aspect of the environment to ensure the correctness of the decision-making. This is especially true for apps that move in various unknown and uncontrolled environment. Building a fit for purpose perception using available data from the environment is one way to simplify the deployment since it alleviates the need to know a priori the peers we need to communicate with. As stated in section 3.2, it is not possible to use data as it is produced; it is then necessary to calibrate the raw data issued from a sensor to take into account its specificities. Such a calibration depends on the usage, this is why it is often done upon a complex installation phase on the field and not during the manufacturing process. Some solutions exist to solve this issue. As an application could not know a priori all sensors, if nobody does it before, it has to establish knowledge about sensors and the quality of the information they generate (eg. how to calibrate them) for a set of purposes. Such 
knowledge could be stored in the environment, then the information could be provided to other applications or instances when needed.

More generally to ease the design of new applications and to align the development of new products with the ever faster standard developments, continuous integration could be used in parallel with continuous conformance and interoperability testing. New shared platforms like [1] aim at facilitating this by providing remote testing tools. Unfortunately, from an application perspective it is not possible to be certain that all potential peers in the surrounding have a compliant behavior for the property it needs [4]. Moreover, upon failure or security breach, a piece of equipment could stop to operate properly and lead to global misbehavior. Testing devices at runtime is then necessary. As for calibration the result of such conformance or interoperability tests could be stored safely in the environment by authoritative testing entities. Applications could then interact with the device with a higher confidence. The confidence level of a device could be part of the quality attribute of the information it contributed to generate.

\section{Conclusion}

We highlighted some of the complexity and the challenges of designing, testing and maintaining pervasive health app that need a great deal of interactions with smart spaces. An illustration based on asthma management highlights the importance of the interactions with the smart space to offer better and more scalable health apps. It then discussed how we envision smarter space eco-systems with a focus on one challenge: providing health app developers with tools to access local environment data. Solving this first challenge would make health apps able to perceive their environment and dynamically adjust their behavior (e.g. level of automation, need for additional information/data) to the actual status of their environment. This, in turn, would create smarter spaces and enable more scalable and reliable health apps.

In this paper, we do not address explicitly the privacy issues. However, our approach is inspired by the fog computing principles and collecting pertinent information and tagging the environment close to the users lead us to consider several questions in relation with data sovereignty/residency: What is the nature of the stored data? Where is it stored? Who holds it? Who can access it? We may consider the models [2] in the design of our tools. In our study case, we partially solve the issues by using citizen participation: Sam, the asthma suffer has the option to choose where his data comes from, where he stores it and who has access to it.

This paper envisioned smarter spaces with massive but scalable interactions between individuals and their everyday working and living environments. The potential of the smarter space is huge and not limited to health app. Smart space could help support better housing facilities, better accessibility for disabled people, smart plant, automated cooperative vehicle or car-Infrastructure cooperation. 


\section{References}

1. F-interop: Platform for online interoperability and performance test. http://www . f-interop.eu/ (2016), [Online; accessed 1-March-2018]

2. Antignac, T., Mtayer, D.L.: Privacy by Design: From Technologies to Architectures. In: Second Annual Privacy Forum, APF 2014. pp. 1-17. Athens, Greece (May 2014)

3. Becker, S., Miron-Shatz, T., Schumacher, N., Krocza, J., Diamantidis, C., Albrecht, U.V.: mhealth 2.0: Experiences, possibilities, and perspectives. JMIR mHealth uHealth 2(2) (May 2014)

4. Calinescu, R., Ghezzi, C., Kwiatkowska, M., Mirandola, R.: Self-adaptive software needs quantitative verification at runtime. Commun. ACM 55(9), 69-77 (Sep 2012)

5. Challa, S., Gulrez, T., Chaczko, Z., Paranesha, T.: A data driven approach for discovering data quality requirements. In: IEEE 8th International Conference on Information Fusion. University of Auckland Business School, Auckland, New Zealand (Dec 2014)

6. Dempster, A.: Upper and lower probabilities induced by a multivalued mapping. Annals of Mathematical Statistics 38, 325-339 (1967)

7. Huang, X., Matricardi, P.M.: Allergy and asthma care in the mobile phone era. Clinical Reviews in Allergy \& Immunology (May 2016)

8. Jayaraman, P.P., Zaslavsky, A., Delsing, J.: On-the-Fly Situation Composition within Smart Spaces. In: Springer (ed.) RUSMART 2011 : 4th International Conference on Smart Space and next generation wired/wireless networking. pp. 52-65. Springer, Saint Petersburg, Russia (Aug 2009)

9. Jayawardene, V., Sadiq, S., Indulska, M.: An Analysis of Data Quality Dimensions. Tech. rep., The University of Queensland (02 2015)

10. Khaleghi, B., Khamis, A., Karray, F.O., Razavi, S.N.: Multisensor data fusion: A review of the state-of-the-art. Information Fusion 14(1), 28 - 44 (2013)

11. Mandl, K., Mandel, J., Kohane, I.: Driving innovation in health systems through an apps-based information economy. Cell Systems 1(1), 8-13 (July 2015)

12. Mun, M., Reddy, S., Shilton, K., Yau, N., Burke, J., Estrin, D., Hansen, M., Howard, E., West, R., Boda, P.: Peir, the personal environmental impact report, as a platform for participatory sensing systems research. In: Proceedings of the 7th International Conference on Mobile Systems, Applications, and Services. pp. 55-68. MobiSys '09, ACM, New York, NY, USA (2009)

13. Pietropaoli, B., Dominici, M., Weis, F.: Multi-sensor data fusion within the belief functions framework - application to smart home services. In: Springer (ed.) RUSMART 2011 : 4th International Conference on Smart Space and next generation wired/wireless networking. pp. 123-134. Springer, Saint Petersburg, Russia (Aug 2011)

14. Shi, W., JieCao, Zhang, Q., Li, Y., Xu, L.: Edge computing: Vision and challenges. IEEE Internet of Things Journal 3(5), 637-646 (October 2016)

15. TalebiFard, P., Leung, V.C.: A data fusion approach to context-aware service delivery in heterogeneous network environment. pp. 312-319. 2nd International Conference on Ambient Systems, Networks and Technologies (ANT-2011), Niagara Falls, Canada (September 2011)

16. Votis, K., Lalos, A., Moustakas, K., Tzovaras, D.: Analysis, modelling and sensing of both physiological and environmental factors for the customized and predictive self-management of asthma. In: 6th Panhellenic Conference of Biomedical Technology. Athens, Greece (May 2015) 